\title{
Morphology and vocalization support specific status of the Chestnut-headed Chachalaca, Ortalis motmot ruficeps (Wagler, 1830) (Aves; Galliformes; Cracidae)
}

\author{
Barbara Mizumo Tomotani ${ }^{1,2,4}$; Luís Fábio Silveira ${ }^{1,5}$ \& José Fernando Pacheco ${ }^{3}$ \\ ${ }^{1}$ Universidade de São Paulo (USP), Museu de Zoologia (MZUSP). São Paulo, SP, Brasil. \\ ${ }^{2}$ Museum of New Zealand Te Papa Tongarewa. Wellington, New Zealand. \\ ${ }^{3}$ Comitê Brasileiro de Registros Ornitológicos (CBRO). Rio de Janeiro, RJ, Brasil. \\ ORCID: http://orcid.org/0000-0002-2399-7662. E-mail: jfpacheco@terra.com.br \\ ${ }^{4}$ ORCID: http://orcid.org/0000-0002-8855-4803. E-mail: babi.mt@gmail.com \\ ${ }^{5}$ ORCID: http://orcid.org/0000-0003-2576-7657.E-mail:Ifs@usp.br
}

\begin{abstract}
The Little Chachalaca (Ortalis motmot) is a widely distributed species in the Amazon basin, typically found in riverine habitats. There are two disjunct populations: the northern 0. m. motmot and the southern 0. m. ruficeps (known as Chestnutheaded (hachalaca). Here we performed a vocal, morphological and plumage comparison between these two taxa. Birds present differences in tail coloration but are otherwise undiagnosable in terms of plumage. Ortalis m. ruficeps is, however, markedly smaller and lighter than $0 . m$. motmot, with no overlap in size or weight. We also found vocal differences between 0. m. motmot and 0 . m. ruficeps, no overlap in geographic distributions and no signs of hybridization across its range. Based on the available data, Ortalis m. ruficeps thus must be considered a valid species, endemic to Brazil.
\end{abstract}

Keywords. Morphology; Plumage; Voice; Little Chachalaca.

\section{INTRODUCTION}

Despite its large size, conspicuousness, ecologic, social and economic importance, the cracids comprise a group of Galliformes of largely neglected taxonomy. The definition of many taxa within this family is presently supported by early $19^{\text {th }}$ century analysis of few specimens and poorly analyzed populations. Despite the relatively large amount of available material in museums, just a handful of studies were performed based on good series of specimens thoroughly covering the species distribution. Unsurprisingly, recent studies using different sets of analytic tools (depending on the available material) have been demonstrating the need of revision of polytypic taxa, in order to discriminate true evolutionary entities from taxonomic rubbish, with the consequent uncovering of valid taxa of immediate conservation concern (e.g., Grau et al., 2003; Silveira et al., 2004; Costa et al., 2017; Evangelista-Vargas et al., 2017; Silveira et al., 2017; Evangelista-Vargas \& Silveira, 2018). For example, the recognition of $O$. remota as a critically endangered valid species (Silveira et al., 2017) promptly ignited a series of field studies directed to the conservation of the few remaining individuals.

Within the cracids, the chachalacas (genus Ortalis Merrem, 1786) exemplify well this taxonomic inflation, with five out of 15 of the accepted species being polytypic (O. vetula, O. ruficauda, O. canicollis, O. guttata and O. motmot; Silveira et al., 2017). The Little Chachalaca, O. motmot (Linnaeus, 1766), currently counts with two recognized subspecies: the nominate O. m. motmot and O. $m$. ruficeps (Wagler, 1830). The latter was originally described as a full species (Penelope ruficeps) by Wagler (1830), based on a specimen from Pará state, northern Brazil. Wagler (1830) proposed that $O$. ruficeps was similar, but much smaller than O. motmot, and had the basal portion of the outer tail feathers colored bronzy brass. Sclater \& Salvin (1870) and Allen (1876) agreed with Wagler (1830, 1832), using the name Ortalida ruficeps. In a subsequent study, Riker \& Chapman (1891) presented $O$. ruficeps under the name Ortalida motmot, which was considered a mistake by Hellmayr \& Conover (1942). Ogilvie-Grant $(1893,1897)$ used the name Ortalis ruficeps mentioning the outer tail feathers blackish glossed with green and tipped 
with chestnut. Ihering \& Ihering (1907) and Heinroth (1922) subsequently kept the species rank of O. ruficeps.

The subordination of O. ruficeps to O. motmot was first proposed by Todd (1932), who analyzed a series of specimens from the lower Amazon. This author mentioned the smaller size and differences in head and tail coloration of $O$. ruficeps but suggested that it only represented a race of $O$. motmot. Peters (1934) agreed to this treatment and all subsequent authors followed suit, without presenting additional analyses (Pinto, 1938; Hellmayr \& Conover, 1942; Pinto, 1964; Vaurie, 1965, 1968; Delacour \& Amadon, 1973; Blake, 1977; Pinto, 1978; del Hoyo et al., 1994; Grantsau, 2010). These studies maintained a similar description and diversely pointed out to various diagnostic features of $O$. ruficeps in comparison with O. motmot: the smaller size (Hellmayr \& Conover, 1942; Pinto, 1964; Delacour \& Amadon, 1973; Grantsau, 2010); a lighter and/or brighter coloration of the crown in O. ruficeps (Hellmayr \& Conover, 1942; Pinto, 1964); a redder coloration of the crown (Hellmayr \& Conover, 1942; Blake, 1977); and a darker overall coloration (Pinto, 1964; Delacour \& Amadon, 1973; Grantsau, 2010). Differences in the color of the external rectrices were also raised (Hellmayr \& Conover, 1942; Blake, 1977), although Vaurie (1965) argued that this was a more subtle character. Sick $(1985,1997)$ was the only author who consistently kept the species rank for O. ruficeps.

Recent studies have shown that a closer examination of large cracids series can reveal several valid species within the subspecies of polytypic complexes (e.g., Ortalis guttata), in some instances with dramatic implications for conservation (Silveira et al., 2017). Therefore, in the present study, we conducted a thorough examination of the available museum skins of O. m. motmot and O. m. ruficeps, together with high quality photographs and vocalizations available in online repositories, in order to provide a taxonomic revision of these two supposed subspecies, with updated diagnoses and geographic distribution.

\section{MATERIAL AND METHODS}

We examined 147 specimens deposited in the collections of the Museu de Zoologia da Universidade de São Paulo (MZUSP, São Paulo, Brazil), American Museum of Natural History (AMNH, New York, USA), Colección Ornitológica Phelps (COP, Caracas, Venezuela), Estación Biológica de Rancho Grande (Aragua, Venezuela), Field Museum of Natural History (FMNH, Chicago, USA), Muséum National d'Histoire Naturelle (MNHN, Paris, France), Museum für Naturkunde (MfN, Berlin, Germany), Naturalis Biodiversity Center (RMNH, Leiden, the Netherlands), and Staatliches Musem für Naturkunde Stuttgart (SMNS, Stuttgart, Germany), and we measured 76 of those specimens (51 O. m. motmot and 25 O. m. ruficeps, including males and females from Venezuela and Brazil deposited at COP and MZUSP, respectively).

We also obtained photographic records from the WikiAves platform (www.wikiaves.com.br) to comple- ment the distribution map (31 localities). Finally, we gathered 40 voice recordings from Xeno-Canto (www. xeno-canto.org) and WikiAves, from which 18 were suitable for spectrographic analyses.

We compared the birds in terms of plumage coloration, morphology and voice. For plumage coloration we analyzed the color of the crown, forehead, throat, chest, abdomen, flanks, crissum, mantle, wing coverts, primaries, tail coverts, external and central rectrices. For morphology we measured the exposed culmen, bill width and depth, and tarsus (nearest $0.01 \mathrm{~mm}$ ) as well as the wing and tail length (nearest $0.1 \mathrm{~cm}$ ). Finally, for the vocal analysis we measured the total syllable duration and the duration and spacing of each note, using the Software Raven Pro 1.5 (Bioacoustics Research Program, 2014).

Statistical analyses were conducted in $\mathrm{R}$ version 3.4.3 (R Core Team, 2017) for morphological and voice characters. We first tested separately whether each of the measurements and vocal characters were explained by taxa, sex and the interaction between taxa and sex (only taxa in the case of the vocal characters). Model selection was performed via stepwise backwards selection, dropping non-significant terms with the critical $p$-value corrected for multiple testing (Bonferroni correction $=0.05 /$ number of tests) in each step. Next, we conducted a Principal Component Analysis on the standardized measurement values (mean centered at 0 , standard error at 1) of the group of morphometry and voice characters. Missing values were replaced by zeros after the standardization (thus replaced by the mean value). Principal components 1 and 2 explained $81 \%$ of the variation for morphometrics and $62 \%$ for voice characters and were retained for subsequent analyses. Lastly, we conducted two analyses: a multivariate analysis of variance (MANOVA) with both PC1 and PC2 as response variables and two separate regressions for $\mathrm{PC} 1$ and PC2 separately (similar to the first analysis), once more with the critical $p$-value corrected for multiple testing.

\section{RESULTS AND DISCUSSION}

As previously reported in the literature (Wagler, 1832; Ogilvie-Grant, 1893, 1897; Hellmayr \& Conover, 1942; Vaurie, 1965; Blake, 1977), O. m. motmot and O. m. ruficeps differ from each other in the coloration of the rectrices, but are identical in other plumage characters, with little intraspecific variation. The border of the external rectrices are tinged with olive dark brown in O. m. ruficeps, while they are reddish brown in 0. m. motmot (Fig. 1).

We did not observe any of the other differences in plumage reported in earlier studies, even after analyzing a large number of specimens. We did observe a lighter crown color in a few specimens of $O$. m. ruficeps (MZUSP $17054,22045,22046,22818,22819,46256)$, as previously reported (Todd, 1932; Hellmayr \& Conover, 1942; Pinto, 1964), but in all other specimens the coloration was identical to O. m. motmot. Moreover, we did not observe differences in darkness or redness in other plumage characters (contra Hellmayr \& Conover, 1942; Blake, 1977; Pinto, 1964; Delacour \& Amadon, 1973; Grantsau, 2010). 
On the other hand, and confirming what was reported in the literature since its original description (Wagler, 1830; Todd, 1932; Hellmayr \& Conover, 1942; Pinto, 1964; Vaurie, 1965; Delacour \& Amadon, 1973; Grantsau, 2010), O. $m$. ruficeps is smaller than $0 . m$. motmot in almost every character analyzed and the differences are maintained even when sexes differ in size. Furthermore, there is no overlap in size range between the two taxa. When compared to O. m. motmot, O. m. ruficeps has a smaller culmen (10\%; percentage difference between brackets), bill width (13\%), tarsus (16\%), wing (12\%), and tail length (16\%; see Appendix 1). Ortalis m. ruficeps is also significantly smaller when using the principal components PC1 and PC2, with males larger than females (Fig. 2, MANOVA: Taxon $F_{270}=197.85, p<0.01$, Sex $F_{269}=6.46, p<0.01$; see also Table 1 and Appendix 2). To complement our morphological analyses, we obtained the fresh mass from the label of ten specimens of O. m. motmot (from Venezuela and Brazil deposited at MZUSP and EBRG) and a single record from the literature for O. m. ruficeps (Graves \& Zusi, 1990). Ortalis m. motmot is heavier than O. m. ruficeps, weighing on average $476 \mathrm{~g}$ (ranging from 431 to $520 \mathrm{~g}$, including males and females), while the sole adult male of O. m. ruficeps (Graves \& Zusi, 1990) weighed 345 g, 27\% lighter than the nominate form. Finally, contrasting to what is reported in the literature (del Hoyo et al., 1994), individuals of $0 . \mathrm{m}$. motmot from Venezuela are not as small as $O$. m. ruficeps.

The vocalizations of $O . m$. ruficeps differ from those of O. m. motmot, but the results should be interpreted with some caution due to the limited sample available for O. m. ruficeps. The main difference of the song of O. m. ruficeps when compared with O. m. motmot is the longer syllable duration (Fig. 3, MANOVA: Taxon $F_{248}=12.61$, $p<0.01)$, although the duration of each note varied among the two taxa (Appendix 3 and 4).
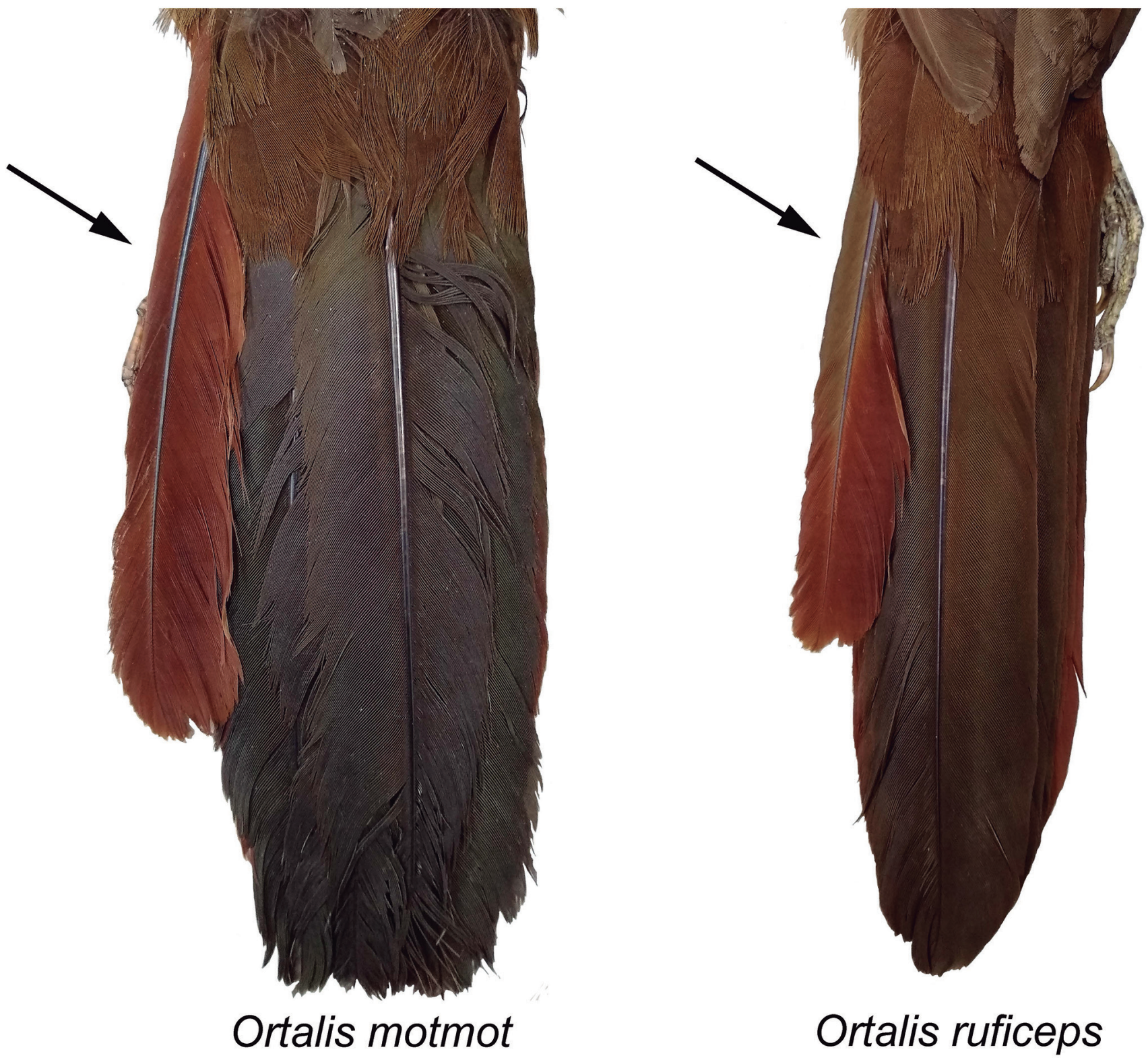

Figure 1. Photographs of the tail of 0 . motmot (MZUSP 82332) and 0. ruficeps (MZUSP 102858) showing the color differences of the external rectrices (arrows). In 0 . ruficeps the border of the external rectrices are tinged with olive dark brown while in 0. motmot they are reddish brown. 

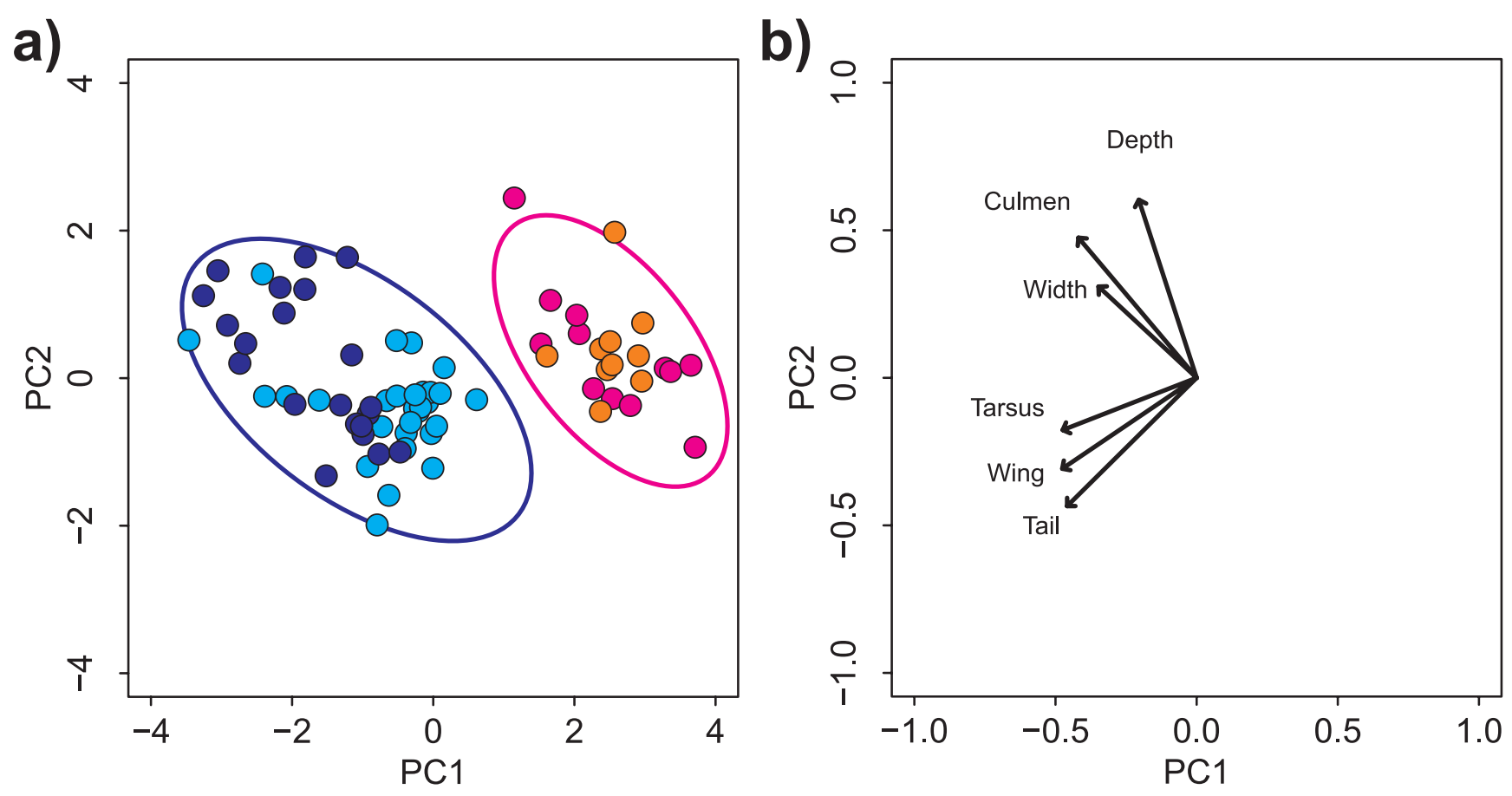

Figure 2. (a) Scatterplot of the two first components obtained from the Principal Component Analysis of the morphometric measurements. Blue: 0 . motmot, Red: 0. ruficeps. Dark-colored symbols - males, light-colored symbols - females. Ellipses are $95 \%$ confidence ellipse (b) Loadings plot of the two first two first components obtained from the Principal Component Analysis of the morphometric measurements.
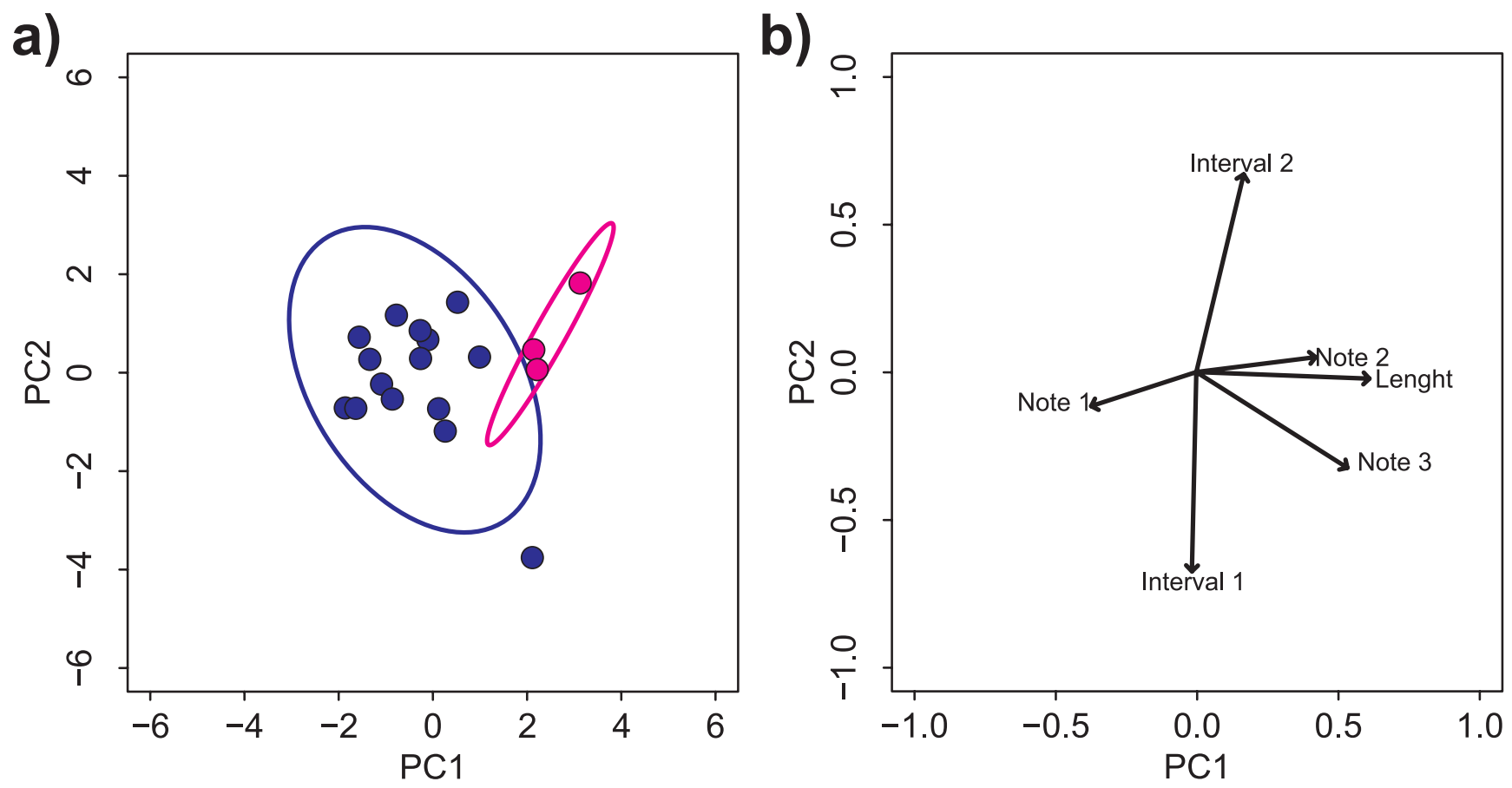

Figure 3. (a) Scatterplot of the two first components obtained from the Principal Component Analysis of the vocal characters. Blue: 0 . motmot, Red: 0 . ruficeps. Ellipses are 95\% confidence ellipse (b) Loadings plot of the two first two first components obtained from the Principal Component Analysis of the vocal characters.

Ortalis m. motmot and O. m. ruficeps are common and locally abundant, being separated by the Amazon River. Populations of $O$. $m$. ruficeps occur south of the river (Pará and Mato Grosso states), following the courses of the rivers Tapajós, Xingu, Tocantins and Araguaia, while the distribution of O. m. motmot extends northward from the Amazon River, including the Brazilian states of Pará, Amazonas, Amapá and Roraima, as well as Guyana, French Guiana, Suriname and Venezuela (Fig. 4).
Although occurring mostly in riverine habitats, there is no sign of contact between northern and southern populations. Thus, these birds comprise another classic case in Amazonian biogeography of taxa separated by the course of the main rivers (Cracraft, 1985). A few specimens of $O$. $m$. ruficeps allegedly share the same localities of O. m. motmot specimens (Lago Cuipeua and Pinhel, both in Pará). These individuals were also noticed by Vaurie (1965), who regarded the localities as erroneous, 
Table 1. Model results for the multiple regression analyses testing the effects of taxon and sex on the various morphometric measurements as well as means and standard errors (s.e.) for each taxon-sex combination. Statistics are given for each term at the point of exclusion of the term from the model. $P$-values in bold with an asterisk represent significance after a Bonferroni correction to account for multiple testing (critical $p$-value $=0.05$ /number of tests).

\begin{tabular}{|c|c|c|c|c|c|c|}
\hline Bill culmen (mm) & Estimate & s.e. & ndf & ddf & $F$-test & $p$-value \\
\hline Taxon: sex & & & 1.00 & 67.00 & 7.79 & $<0.01^{*}$ \\
\hline Taxon (0. ruficeps): Sex (male) & -2.00 & 0.72 & & & & \\
\hline Taxon (0. motmot) & 22.70 & 0.26 & & & & \\
\hline Taxon (0. ruficeps) & 21.26 & 0.44 & & & & \\
\hline Sex (male) & 1.42 & 0.40 & & & & \\
\hline Bill width (mm) & Estimate & s.e. & ndf & ddf & $F$-test & $p$-value \\
\hline Taxon : sex & & & 1.00 & 48.00 & 4.5199 & 0.04 \\
\hline Taxon & & & 1.00 & 50.00 & 39.33 & $<0.01^{*}$ \\
\hline Sex & & & 1.00 & 49.00 & 0.17 & 0.68 \\
\hline Taxon (0. ruficeps): Sex (male) & -0.76 & 0.36 & & & & \\
\hline Taxon (0. motmot) & 9.16 & 0.12 & & & & \\
\hline Taxon (0. ruficeps) & 8.00 & 0.14 & & & & \\
\hline Sex (male) & 0.08 & 0.18 & & & & \\
\hline Bill depth (mm) & Estimate & s.e. & ndf & ddf & $F$-test & $p$-value \\
\hline Taxon : sex & & & 1.00 & 45.00 & 1.16 & 0.29 \\
\hline Taxon & & & 1.00 & 46.00 & 5.76 & 0.02 \\
\hline Sex & & & 1.00 & 46.00 & 12.69 & $<0.01^{*}$ \\
\hline Taxon (0. ruficeps): Sex (male) & -0.44 & 0.41 & & & & \\
\hline Taxon (0. motmot) & 9.49 & 0.16 & & & & \\
\hline Taxon (0. ruficeps) & 9.00 & 0.19 & & & & \\
\hline Sex (male) & 0.71 & 0.20 & & & & \\
\hline Tarsus $(\mathrm{cm})$ & Estimate & s.e. & ndf & ddf & $F$-test & $p$-value \\
\hline Taxon: sex & & & 1.00 & 67.00 & 0.2958 & 0.59 \\
\hline Taxon & & & 1.00 & 68.00 & 200.56 & $<0.01^{*}$ \\
\hline Sex & & & 1.00 & 68.00 & 10.28 & $<0.01^{*}$ \\
\hline Taxon (0. ruficeps): Sex (male) & -0.75 & 1.39 & & & & \\
\hline Taxon (0. motmot) & 57.25 & 0.47 & & & & \\
\hline Taxon (0. ruficeps) & 47.49 & 0.67 & & & & \\
\hline Sex (male) & 2.05 & 0.64 & & & & \\
\hline Wing $(\mathrm{cm})$ & Estimate & s.e. & ndf & ddf & $F$-test & $p$-value \\
\hline Taxon: sex & & & 1.00 & 69.00 & 1.15 & 0.29 \\
\hline Taxon & & & 1.00 & 71.00 & 250.87 & $<0.01^{*}$ \\
\hline Sex & & & 1.00 & 70.00 & 6.19 & 0.02 \\
\hline Taxon (0. ruficeps): Sex (male) & -0.31 & 0.29 & & & & \\
\hline Taxon (0. motmot) & 19.94 & 0.08 & & & & \\
\hline Taxon (0. ruficeps) & 17.57 & 0.12 & & & & \\
\hline Sex (male) & 0.33 & 0.13 & & & & \\
\hline $\begin{array}{r}\text { Tail }(\mathrm{cm}) \\
\end{array}$ & Estimate & s.e. & ndf & ddf & $F$-test & $p$-value \\
\hline Taxon: sex & & & 1.00 & 69.00 & 0.93 & 0.34 \\
\hline Taxon & & & 1.00 & 71.00 & 191.85 & $<0.01^{*}$ \\
\hline Sex & & & 1.00 & 70.00 & 2.80 & 0.10 \\
\hline Taxon (0. ruficeps): Sex (male) & -0.53 & 0.55 & & & & \\
\hline Taxon (0. motmot) & 24.52 & 0.15 & & & & \\
\hline Taxon (0. ruficeps) & 20.66 & 0.23 & & & & \\
\hline Sex (male) & 0.43 & 0.25 & & & & \\
\hline PC1 & Estimate & s.e. & ndf & ddf & F-test & $p$-value \\
\hline Taxon: sex & & & 1.00 & 69.00 & 5.75 & 0.02 \\
\hline Taxon & & & 1.00 & 70.00 & 281.83 & $<0.01^{*}$ \\
\hline Sex & & & 1.00 & 70.00 & 13.10 & $<0.01^{*}$ \\
\hline Taxon (0. ruficeps): Sex (male) & 1.02 & 0.42 & & & & \\
\hline Taxon (0. motmot) & -0.77 & 0.15 & & & & \\
\hline Taxon (0. ruficeps) & 2.91 & 0.21 & & & & \\
\hline Sex (male) & -0.73 & 0.20 & & & & \\
\hline PC2 & Estimate & s.e. & ndf & ddf & $F$-test & $p$-value \\
\hline Taxon: sex & & & 1.00 & 69.00 & 2.18 & 0.14 \\
\hline Taxon & & & 1.00 & 70.00 & 3.56 & 0.06 \\
\hline Sex & & & 1.00 & 71.00 & 4.33 & 0.04 \\
\hline Taxon (0. ruficeps): Sex (male) & -0.60 & 0.41 & & & & \\
\hline Taxon (0. motmot) & -0.19 & 0.13 & & & & \\
\hline Taxon (0. ruficeps) & 0.22 & 0.14 & & & & \\
\hline Sex (male) & 0.36 & 0.19 & & & & \\
\hline$P C 1$ and $P C 2$ (MANOVA) & Residuals & Pillai & ndf & ddf & $F$-test & $p$-value \\
\hline Taxon: sex & 69.00 & 0.08 & 2 & 68 & 2.89 & 0.06 \\
\hline Taxon & 70.00 & 0.85 & 2 & 70 & 197.85 & $<0.01 *$ \\
\hline Sex & 71.00 & 0.16 & 2 & 69 & 6.456 & $<0.01^{*}$ \\
\hline
\end{tabular}

which was later supported by Pinto (1978). These localities were also scrutinized by Wiley (2010), who raised a considerable suspicion of carelessness in labeling by the original collector A. Olalla. Thus, the correct provenance of these specimens is considered doubtful. Because of this and the lack of additional material indicating syntopy, we excluded these problematic localities from the map (Fig. 4).

Although uncommon in the taxonomy of recent birds, differences in dimensions of distinct and unrelated morphological traits are a strong indicative of the existence of distinct evolutionary lineages. Our expertise with osteology of Cracids (see Silveira, 2003; Grau et al., 2005; Frank-Hoeflich et al., 2007) show that modifications in skeleton and body size seem to evolve at a slower pace than plumage and voice, and finding these osteological differences, without overlap, strongly support the existence of two distinct species. Bone measurements and size differences are widely used and supported in paleontological studies to delineate species (e.g., Olson 1974; Balouet \& Olson 1987; Alvarenga et al., 2002; Alvarenga \& Olson, 2004; Mayr et al., 2017), as well as in neontology to validate taxa with disjunct populations (Alvarenga et al., 2002). Our extensive analysis of O. m. motmot and O. $m$. ruficeps show that the population south of the Amazon River differs considerably in size from birds from north of the Amazon, without overlap; differences in plumage and voice are also present, though of smaller magnitude. Based on these differences (size, plumage, and voice), coupled with the distinct geographical distribution, we therefore argue that Ortalis ruficeps (Wagler, 1830) should be treated as a valid species, distinct from O. motmot, as originally described and presented below.

\section{Systematics \\ Order Galliformes Family Cracidae \\ Genus Ortalis Merrem, 1786 Ortalis motmot (Linnaeus, 1766)}

Phasianus motmot Linnaeus, 1766: 271.

Phasianus katraca Boddaert, 1783: 9.

Phasianus Parraka Gmelin, 1789: 740.

Phasianus Parraqua Latham, 1790: 632.

Phasianus Parrakoua Temminck, 1815: 695.

Penelope motmot Wagler, 1830: 1111.

Penelope paraca Schomburgk, 1848: 31.

Ortalida motmot Cabanis, 1849: 744; Sclater \& Salvin, 1870: 532; Pelzeln, 1870: 285, 1873: 119; Brown, 1876: 373; Goeldi, 1897: 161.

Penelope Parrakua: Burmeister, 1856: 341.

Ortalis motmot: Salvin, 1886: 175; Ogilvie-Grant, 1893: 505, 1897: 235; Goeldi, 1897: 156; Berlepsch \& Hartert, 1902: 120; Hellmayr, 1907: 39; Berlepsch, 1908: 297; Snethlage, 1914: 56; Cherrie, 1916: 356; Chubb, 1916: 27; Beebe et al., 1917: 127; Bangs \& Penard, 1918: 40; Chapman, 1931: 61; Sick, 1985: 232; Sick, 1997, 2001: 277. 


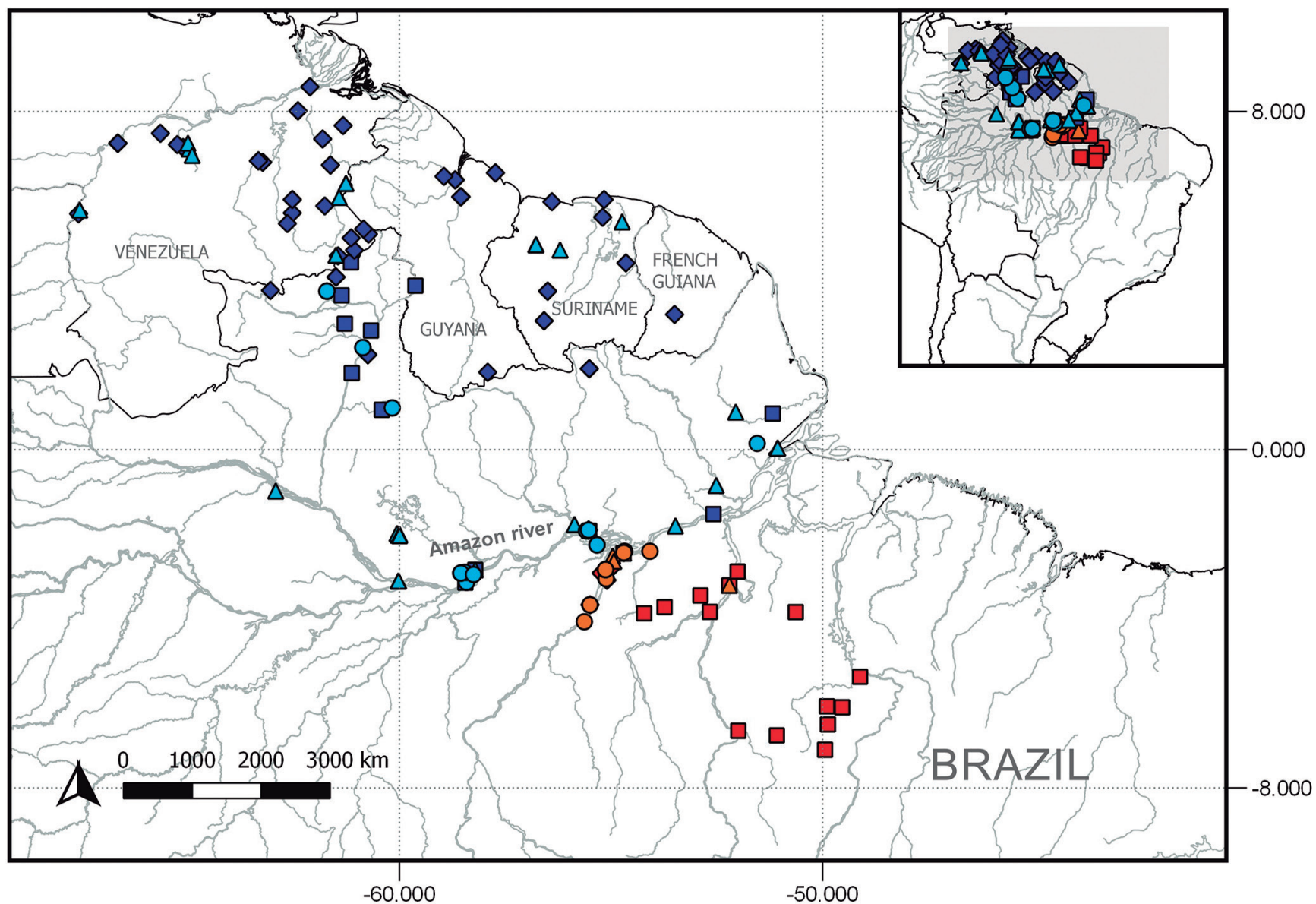

Figure 4. Distribution of 0 . motmot (blue and light blue) and of 0 . ruficeps (orange and red). Circles: measured specimens; triangles: vocalizations; diamonds: skins not measured; squares: photographs.

Ortalis katraca: Ihering \& Ihering, 1907: 15.

Ortalis motmot motmot: Peters, 1934: 17; Hellmayr \& Conover, 1942: 161; Pinto, 1964: 107; Vaurie, 1965: 19, 1968: 244; Delacour \& Amadon, 1973: 116; Blake, 1977: 396; Pinto, 1978: 78; del Hoyo et al., 1994: 345; Grantsau, 2010: 63.

Type material: The species was described based on a specimen deposited in $M$. Réaumur's collection. Specimens from his collection eventually came to MNHN, but we were unable to locate an Ortalis motmot with compatible data in the MNHN collection, and we assume it is lost. See also Voisin et al. (2015).

Type locality: Cayenne, French Guiana.

Description: No sexual dimorphism in plumage color. Crown dark reddish brown. Forehead dark reddish brown. Bill gray. Throat yellowish red. Chest dark grayish brown. Abdomen, flanks and crissum pale brown. Mantle dark yellowish brown. Wing primaries and coverts dark brown. Tail coverts dark reddish brown. External rectrices dark reddish brown. Central rectrices dark brown. Males larger than females.

Measurements: See Table 1.

Voice: See Table 2.
Diagnosis: Similar to O. ruficeps, but border of external rectrices redder; size considerably larger (with no overlap); different vocalization (shorter syllable length, and consequently shorter song).

Distribution: Venezuela, Guyana, French Guiana, Suriname, and northern Brazil (Pará, Roraima, Amapá and Amazonas states).

Specimens examined ( $\mathbf{n}=\mathbf{1 1 1})$ : BRAZIL: Amazonas: Comunidade Vila Nova, Macapá (MZUSP 82332); Rio Amazonas, Lago de Camaçari (MZUSP 22108, 22064, 22284); Rio Amazonas, Itacoatiara (MZUSP 22130, 22178); Rio Amazonas, Silves (MZUSP 22175, 22177, 22181, 21420); Rio Amazonas, Igarapé Anibá (MZUSP 21092, 22017, 22107, 22122, 22817). Pará: Rio Amazonas, Boca do canal Piava, 10 mi E Óbidos (FMNH 410726, 410727); Rio Trombetas, Jacaré (MZUSP 59839, 59840); Rio Amazonas, Jacuara (FMNH 422549); Lago Cuipeva (FMNH 410722, 410723, 410724, 410725); Lago Grande (MZUSP 10599, 10600, 10601); Rio Amazonas, Lago Cuiteuá (MZUSP 15693, 15694); Rio Amazonas, Igarapé Buiussú (MZUSP 22134). Roraima: BR-174, km 709 (MZUSP 81386), Rio Mucajaí (MZUSP 55750); Rio Jufari, Sitio Valdir (MZUSP 93582); Rio Mucajaí, São João da Boa Vista (MZUSP 55751); Serra da Lua, near Boa Vista (FMNH 46870, 46871, 46872, 46873); Serra Tepequém, V. Cabo Sobral, Amajari (MZUSP 79080, 79081, 79082). FRENCH GUIANA: Camopi (MNHN 
Table 2. Model results for the simple regression analyses testing the effects of taxon on the vocal characters, as well as means and standard errors for each taxon. Statistics are given for each term at the point of exclusion of the term from the model. $P$-values in bold with an asterisk represent significance after a Bonferroni correction to account for multiple testing (critical $p$-value $=0.05 /$ number of tests).

\begin{tabular}{|c|c|c|c|c|c|c|}
\hline Total duration & & & ndf & ddf & $F$-test & $p$-value \\
\hline Taxon & & & 1.00 & 16.00 & 10.33 & $<0.01^{*}$ \\
\hline & mean & s.e. & & & & \\
\hline 0.m. motmot & 0.47 & 0.01 & & & & \\
\hline 0.m. ruficeps & 0.54 & 0.01 & & & & \\
\hline Note 1 & & & ndf & ddf & $F$-test & $p$-value \\
\hline Taxon & & & 1.00 & 16.00 & 3.18 & 0.09 \\
\hline & mean & s.e. & & & & \\
\hline 0. m. motmot & 0.11 & 0.00 & & & & \\
\hline 0.m. ruficeps & 0.09 & 0.01 & & & & \\
\hline Note 2 & & & ndf & ddf & $F$-test & $p$-value \\
\hline Taxon & & & 1.00 & 16.00 & 14.80 & $<0.01^{*}$ \\
\hline & mean & s.e. & & & & \\
\hline 0. m. motmot & 0.12 & 0.01 & & & & \\
\hline 0.m. ruficeps & 0.16 & 0.01 & & & & \\
\hline Note 3 & & & ndf & ddf & $F$-test & $p$-value \\
\hline Taxon & & & 1.00 & 16.00 & 3.38 & 0.08 \\
\hline & mean & s.e. & & & & \\
\hline 0.m. motmot & 0.14 & 0.01 & & & & \\
\hline 0.m. ruficeps & 0.17 & 0.01 & & & & \\
\hline Interval 1 & & & ndf & ddf & $F$-test & $p$-value \\
\hline Taxon & & & 1.00 & 16.00 & 2.17 & 0.16 \\
\hline & mean & s.e. & & & & \\
\hline 0. m. motmot & 0.03 & 0.00 & & & & \\
\hline 0.m. ruficeps & 0.02 & 0.01 & & & & \\
\hline Interval 2 & & & ndf & ddf & $F$-test & $p$-value \\
\hline Taxon & & & 1.00 & 16.00 & 1.39 & 0.25 \\
\hline & mean & s.e. & & & & \\
\hline 0. m. motmot & 0.08 & 0.01 & & & & \\
\hline 0.m. ruficeps & 0.10 & 0.01 & & & & \\
\hline PC1 & & & ndf & ddf & $F$-test & $p$-value \\
\hline Taxon & & & 1.00 & 16.00 & 19.20 & $<0.01^{*}$ \\
\hline & mean & s.e. & & & & \\
\hline 0. m. motmot & -0.48 & 0.28 & & & & \\
\hline 0.m. ruficeps & 2.39 & 0.32 & & & & \\
\hline PC2 & & & ndf & ddf & $F$-test & $p$-value \\
\hline Taxon & & & 1.00 & 16.00 & 1.42 & 0.25 \\
\hline & mean & s.e. & & & & \\
\hline 0. m. motmot & -0.15 & 0.32 & & & & \\
\hline 0.m. ruficeps & 0.76 & 0.53 & & & & \\
\hline
\end{tabular}

829); Saut Macaque (MNHN 757). GUYANA: Itabu Creek, Middle Base Camp (FMNH 120033); Rockstone (FMNH 105553); Rockstone, Essequibo River (FMNH 108052, 108053, 108054, 108055, 108056, 413176, 413177, 413178); Abary River (FMNH 190562, 190563); Kartabo Point (FMNH 408015, 408016, 408017); Oko Mts (FMNH 413179); Mahacai Creek (FMNH 32452, 32453); Trois Sauts (MNH 830). SURINAME: Savana Awarra - Wageningen (RMNH 65967-12); Lely Gebergte (RMNH 80449-13); Kayser Gebergte Airstrip, Zuid River (FMNH 422919, 422920); Wilhelmina Gebergte, West River (FMNH 423278, 423279); Paramaribo, vicinity (FMNH 47305); Republiek (RMNH 37980), Unknown locality (SMNS 2066). VENEZUELA: Caño
Cataniapo Atures (COP 20613, 20614); Sierra Pacaraima, Cerro Urutani (AMNH 812850, 812851, COP 73499); Arabapu Mt. Roraima (COP 4064, 4065); Caño Maniapure (EBRG 3618, 3619, 4300, 5665, 6578, 7421, 7520); Base Cerro Uaipan-Tepui (COP 42762); Campo Uriman Rio Caroni (COP 20614); Cerro Tigre Rio Chiguás (COP 15787); El Dorado (COP 17431, EBRG 2108, 2109); Falda, Cerro Cuquenan (COP 50112); Hato Sta. Tereza Rio Uairen (COP 44401); La Faísca Cerro Paurai-Tepui (COP 32257); La Faísca Mirra Cerro Paurai-Tepui (COP 32236); La Paragua (COP 15783, 15784, 15786); Mt. Auyan Tepui (COP 7855, 7856); Peso Callao (COP 74269); Ptari-Tepui Falda Sudoeste (COP 26862); Puerto Carretico Mato Caura (COP 51979); Reserva Florestal El Caura (EBRG 10618); San Ignacio de Yuruani (EBRG 8648); Sierra Imataca Altiplanicie de Nuria (COP 16814); Sta. Rosalia Depto. Cedeño (COP 24983); Upata (EBRG 3985, 3996); Piacoa (FMNH 81436, 81437).

Photographs ( $\mathbf{n}=\mathbf{2 8 7})$ :BRAZIL:Amazonas: Itacoatiara (1); Manaus (148); Presidente Figueiredo (53). Amapá: Ferreira Gomes (3); Macapá (4); Serra do Navio (5). Pará: Almeirim (2); Óbidos (2); Oriximiná (9);Prainha (1). Roraima: Alto Alegre (1); Amajari (42); Boa Vista (2); Caracaraí (7); Normandia (1); Pacaraima (1); Rorainópolis (2).

Voice samples. Used ( $\mathbf{n}=\mathbf{1 5})$ : BRAZIL: Amazonas: Manaus (WA1119199, WA394390); Presidente Figueiredo (XC76224); Silves (WA1043482). Amapá: Macapá (WA135794, WA195670). Pará: Oriximiná (WA1797851); Prainha (WA583167). SURINAME: Foengoe Island (XC172162); Mozeskreek (XC272216, XC272217). VENEZUELA: Bolivar (XC226986); Caura (XC103386, XC226988); La Escalera (XC354952). Not used (n = 20): BRAZIL: Amazonas: Barcelos (WA23908); Manaus (WA154684, WA2063779, WA285768); Presidente Figueiredo (XC13320, XC227403, XC345471). Amapá: Laranjal do Jari (WA1725565); Macapá (WA148965) Serra do Navio (XC85264). FRENCH GUIANA: Armontabo (XC11783). SURINAME: Mapane (XC1065); Mozeskreek (XC272182, XC272183, XC272218). VENEZUELA: Bolívar (XC226985, XC227406, XC227407); Maripa (XC9925); Puerto Ayacucho (XC107593).

\section{Ortalis ruficeps (Wagler, 1830)}

Penelope ruficeps Wagler, 1830: 1111.

Ortalida ruficeps: Wagler, 1832: 1227; Sclater \& Salvin, 1870: 533; Allen, 1876: 82.

Ortalida motmot: Riker \& Chapman, 1891: 162.

Ortalis ruficeps: Ogilvie-Grant, 1893: 504, 1897: 236; Ihering \& Ihering, 1907: 15; Sick, 1985: 232; Sick, 1997; 2001: 277.

Ortalis motmot ruficeps: Todd, 1932: 212; Peters, 1934: 17; Pinto, 1938: 100; Hellmayr \& Conover, 1942: 161; Pinto, 1964: 108; Vaurie, 1965: 16, 1968: 244; Delacour \& Amadon, 1973: 116; Blake, 1977: 396; Pinto, 1978: 78; del Hoyo et al., 1994: 345; Grantsau, 2010: 63.

Type specimen: MfN 11909, examined. 
Type locality: Brazil, Pará state. A more precise locality was not given in the original description, but Erwin Stresemann (in Todd, 1932) argued that the collection site was probably around Cametá, Tocantins River, Pará state.

Description: No sexual dimorphism in plumage color. Crown dark reddish brown, lighter in a few specimens. Forehead dark reddish brown. Bill gray. Throat yellowish red. Chest dark grayish brown. Abdomen, flanks and crissum pale brown. Mantle dark yellowish brown. Wing primaries and coverts dark brown. Tail coverts dark reddish brown. External rectrices dark reddish brown with borders tinged dark brown. Central rectrices dark brown. Males larger than females.

Measurements: See Table 1.

Voice: See Table 2.

Diagnosis: Similar to O. motmot, but border of external rectrices less red; size distinctively smaller (with no overlap); different vocalization (longer syllable length, and consequently slower song).

Distribution: Brazil, south of Amazon River (Pará and Mato Grosso states).

Specimens examined ( $\mathbf{n}=\mathbf{3 6}$ ): BRAZIL: Pará: RioTapajós, Caxiricatuba (FMNH 410096, 410097); Rio Tapajós, Fordlândia (FMNH 422550, 422551; MZUSP 46266, 58096, 68851, 62591, 73244, 102858; Lago Cuipeua (FMNH 418533); Rio Amazonas, Foz do Rio Curuá (MZUSP 17054); Rio Tapajós (MZUSP 67928); Rio Tapajós, Pinhel (FMNH 411103, 411104, 411105); Rio Tapajós, Tauary (FMNH 410099, 410100); Rio Tapajós, Monte Cristo (MZUSP 46265); Rio Tapajós, Piquiatuba (MZUSP 22124, 22282, 22818); Rio Tapajós, Caxiricatuba (MZUSP 17053, 22045, 22046, 22088, 22103, 22123, 22125, 22126, 22131, 22132, 22133, 22819); Rio Tapajós, Santarém (FMNH 410098, MZUSP 46267).

Photographs ( $\mathbf{n}=\mathbf{1 2 5}$ ): BRAZIL: Pará: Altamira (9); Canaã dos Carajás (1); Curionópolis (3); Marabá (1); Medicilândia (1); Ourilândia do Norte (1); Pacajá (1); Parauapebas (19); Placas (1); Santarém (9); São Félix do Xingu (3); Uruará (1); Vitória do Xingu (7); Xinguara (5).

Voice samples. Used ( $\mathbf{n}=3$ ): Pará: Belterra (WA757077); Santarém (XC312921, XC95103). Not used (n= 2): Pará: Altamira (WA1629138); Santarém (WA2288948).

\section{ACKNOWLEDGEMENTS}

We thank the curators and staff of the above listed institutions who kindly received us and allowed us to access the specimens under their care and Conselho Nacional de Desenvovimento Científico e Tecnológico for providing research grants to L.F. Silveira (CNPq \# 302291/2015-6 and \# 457444/2012-6). We also thank to Drs. Wilson Lemos de Moraes Neto (Fazenda Fartura) and Bruno Ehlers (UPS) for support in laboratory and field activities. Finally, we thank the anonymous reviewers for their comments that helped us to improve this manuscript.

\section{REFERENCES}

Allen, J.A. 1876. List of Birds collected by Charles Linden, near Santarem, Brazil. Bulletin of the Essex Institute, 8: 78-83.

Alvarenga, H.M. \& Olson, S.L. 2004. A new genus of tiny condor from the Pleistocene of Brazil (Aves: Vulturidae). Proceedings of the Biological Society of Washington, 117(1): 1-9.

Alvarenga, H.M.; Höfling, E. \& Silveira, L.F. 2002. Notharchus swainsoni (Gray, 1846) (Bucconidae) é uma espécie válida. Ararajuba, 10: 73-77.

Balouet, J.C. \& Olson, S.L. 1987. A new extinct species of giant pigeon (Columbidae: Ducula) from archeological deposits on Wallis (Uvea) Island, South Pacific. Proceedings of the Biological Society of Washington, 100(4): 769-775.

Bangs, 0. \& Penard, T.E. 1918. Notes on a collection of Surinam birds. Bulletin of the Museum of Comparative Zoology, 62(2): 25-93.

Beebe, W.; Hartley, G.I. \& Howes, P.G. 1917. Tropical wild life in British Guiana: zoological contributions from the Tropical Research Station of the New York Zoological Society. New York, New York, Zoological Society. v. 1, 504p.

Berlepsch, H. 1908. Birds of Cayenne. Novitates Zoologicae, 15: 261-324.

Berlepsch, H.V. \& Hartert, E. 1902. On the birds of the Orinoco region. Novitates Zoologicae, 9: 1-134.

Bioacoustics Research Program. 2014. Raven Pro: Interactive Sound Analysis Software (Version 1.5) [Computer software]. Ithaca, The Cornell Lab of Ornithology. Available at: http://www.birds.cornell.edu/raven.

Blake, E.R. 1977. Manual of neotropical birds. V. 1. Spheniscidae (penguins tp Laridae (gulls and allies). Chicago, University of Chicago Press. 674p.

Boddaert, P. 1783. Table des planches enluminéez d'histoire naturelle de M. D'Aubenton : avec les denominations de M.M. de Buffon, Brisson, Edwards, Linnaeus et Latham, precedé d'une notice des principaux ouvrages zoologiques enluminés. Utrecht. 58p.

Brown, C.B. 1876. Canoe and Camp Life in British Guiana. London. 478p.

Burmeister, H. 1856. Systematische Uebersicht der Thiere Brasiliens: welche während einer Reise durch die Provinzen von Rio de Janeiro und Minas geraës gesammlt oder beobachtet Wurden. Vol. 3. Vögel (Aves). Berlin, Georg Reimer. 486p.

Cabanis, J. 1849. Versuch nebst einer zusammenstellung Fauna und Flora Guiana in Britisch-Guiana. In: Schomburgk, R. Reisen in Britisch-Guiana in den Jahren 1840-1844nach Vorlagen von Johannes Müller, Ehrenberg, Erichson, Klotzsch, Troschel, Cabanis und Andern. Leipsig, J.J. Weber. v. 3, p. 534-1260.

Chapman, F.M. 1931. The upper zonal bird-life of Mts. Roraima and Duida. Bulletin of the American Museum of Natural History, v. 63(1): 1-142.

Cherrie, G.K. 1916. A contribution to the ornithology of the Orinoco region. Brooklyn Institute of Art and Sciences, Science Bulletin, 2: 133-374.

Chubb, C. 1921. The birds of British Guiana: based on the collection of Frederick Vavasour McConnell. London, B. Quaritch. v. 2, 615p.

Costa, M.C.; Oliveira Jr., J.R.R.; Davanço, P.V.; Camargo, C.; Laganaro, N.M.; Azeredo, R.M.A.; Simpson, J.G.P.; Silveira, L.F. \& Francisco, M.R. 2017. Recovering the Genetic Identity of an Extinct-in-the-Wild Species: The Puzzling Case of the Alagoas Curassow. Plos One, 12: 1-18.

Cracraft, J. 1985. Historical biogeography and patterns of differentiation within the South American avifauna: areas of endemism. Ornithological Monographs, 36: 49-84.

del Hoyo, J.; Elliot, A. \& Sargatal, J. 1994. Handbook of the birds of the world. Vol. 2. New world vultures to guineafowl. Barcelona, Lynx Edicions. 638p. 
Delacour, J. \& Amadon, D. 1973. Curassows and related birds. New York, American Museum of Natural History. 247p.

Evangelista-Vargas, D.; Costa, T.V.V.; Whitney, B.M.; Schunck, F. \& Silveira, L.F. 2017. Penelope superciliaris pseudonyma Neumann, 1933 (Aves, (racidae) is the valid name for the blue-faced population of Rustymargined Guan endemic to the Madeira-Tapajós interfluvium of central Amazonian Brazil. Zootaxa, 4294: 436-442.

Evangelista-Vargas, 0.D. \& Silveira, L.F. 2018. Morphological evidence for the taxonomic status of the Bridge's Guan, Penelope bridgesi, with comments on the validity of $P$. obscura bronzina (Aves: Cracidae). Zoologia, Curitiba, 35: 1-10, e12993.

Frank-Hoeflich, K.; Silveira, L.F.; Estudillo-López, J.; García-Koch, A.M.; Ongay-Larios, L. \& Piñero, D. 2007. Increased taxon and character sampling reveals novel intergeneric relationships in the Cracidae (Aves: Galliformes). Journal of Zoological Systematics and Evolutionary Research, 45(3): 242-254.

Gmelin, J F. 1789. Systema naturae per regna tria naturae, secundum classes, ordines, genera, species, cum characteribus, differentiis, synonymis, locis. 13. ed. Lipsiae, G. e Beer.

Goeldi, E.A. 1897. Ornithological results of a naturalist's visit to the coastregion of South Guyana. Ibis, 39(2): 149-165.

Grantsau, R.K.H. 2010. Guia completo para identificação das Aves do Brasil. São Carlos, Vento Verde.

Grau, E.T.; Pereira, S.L.; Silveira, L.F. \& Wajntal, A. 2003. Molecular markers contribute to a breeding programme of the extinct-in-the-wild Alagoas Curassow Mitu mitu and confirm the validity of the species. Bird Conservation International, 13: 115-126.

Grau, E.T.; Pereira, S.L.; Silveira, L.F.; Höfling, E. \& Wajntal, A. 2005. Molecular phylogenetics and biogeography of Neotropical piping guans (Aves: Galliformes): Pipile Bonaparte, 1856 is synonym of Aburria Reichenbach, 1853. Molecular Phylogenetics and Evolution, 35: 637-645.

Graves, G.R. \& Zusi, R.L. 1990. Avian body weights from the lower Rio Xingu, Brazil. Bulletin of the British Ornithologists' Club, 110(1): 20-25.

Heinroth. 1922. Bericht über die Festsitzung zur Feier des 70. Geburtstages des Herrn Herman Schalow. Journal für Ornithologie, 70: 492-506.

Hellmayr, C.E. 1907. Another contribution to the ornithology of the Lower Amazonas. Novitates Zoologicae, 14: 1-39.

Hellmayr, C.E. \& Conover, H.B. 1942. Catalogue of Birds of the Americas and the adjacent islands in Field Museum of Natural History. Field Museum of Natural History, Zoological Series, 13(1): 1-636.

Ihering, H. von \& Ihering, R. von 1907. As aves do Brazil. São Paulo, Typ. Diario Oficial. 485p. ( Catalogos da Fauna Brazileira, v. 1)

Latham, J. 1790. Index ornithologicus sive systema ornithologiae: complectens avium divisionem in classes, ordines, genera, species. Londini. v. 1, 466 p.

Linnaeus, C. 1766. Systema naturae per regna tria naturae, secundum classes, ordines, genera, species, cum characteribus, differentiis, synonymis, locis. Tomus I. Editio duodecima, reformata. Stockholm,Laurentii Salvii. 532p.

Mayr, G.; Scofield, R.P.; Pietri, V.L.D. \& Tennyson, A.J.D. 2017. A Paleocene penguin from New Zealand substantiates multiple origins of gigantism in fossil Sphenisciformes. Nature Communications, 1927: 1-8.

Ogilvie-Grant, W.R. 1893. Catalogue of Game Birds. Pterocletes, Gallinae, Ophistocomi, Hemipodii. In: Catalogue of the Birds in the British Museum. London, Order of the Trustees. v. 22, 588p.

Ogilvie-Grant, W.R. 1897. A hand-book to the game-birds. London, WH Allen \& Co.

Olson, S.L. 1974. A new species of Nesotrochis from Hispaniola, with notes on other fossil rails from the West Indies (Aves: Rallidae). Proceedings of the Biological Society of Washington, 87(38): 439-450.

Pelzeln, A. 1870. Zur Ornithologie Brasiliens. Resultate von Johann Natterers Reisen in den Jahren 1817 bis 1835. Wien, Druck und Verlag von A. Pichler's Witwe \& Sohn. 462p.
Pelzeln, A. 1873. Verzeichniss einer an Dr. L.W. Schaufuss gelangten sending Vögel aus Neu-Freiburg in Brasilien.

Peters, J.L. 1934. Check-List of Birds of the World. Cambridge, Harvard University Press. v. 2, 401p.

Pinto, 0.M.0. 1938. Catálogo das aves do Brazil e lista dos exemplares que as representam no Museu Paulista. Revista do Museu Paulista, 22: 1-56.

Pinto, 0.M.0. 1964. Ornitologia Brasiliense: Catalogo descritivo e ilustrado das aves do Brasil. Vol. 1. Parte introdutória e familias Rheidae a Cuculidae. São Paulo, Departamento de Zoologia da Secretaria de Agricultura do Estado de São Paulo.

Pinto, 0.M.0. 1978. Novo catálogo das aves do Brasil, primeira parte-Aves não Passeriformes e Passeriformes não Oscines, com exclusão da família Tyrannidae. São Paulo, Empresa Gráfica da Revista dos Tribunais.

R Core Team. 2017. R: A Language and Environment for Statistical Computing. Vienna, R Foundation for Statistical Computing. Available at: http:// www.R-project.org.

Riker, C.B. \& Chapman, F.M. 1891. A List of Birds Observed at Santarem, Brazil. The Auk, 8: 158-164.

Salvin, 0. 1886. A List of the Birds obtained by Mr. Henry Whitely in British Guiana. Ibis, 28(4): 499-510.

Schomburgk, M.R. 1848. Reisen in Britisch-Guiana in den jahren 1840-1844. Leipzig, J.J. Weber. v. 2.

Sclater, P.L. \& Salvin, 0. 1870. Synopsis of the Cracidae. Proceedings of Zoological Society of London, (1870): 504-544.

Sick, H. 1985. Ornitologia Brasileira: uma introdução. Brasília, Universidade de Brasília. v. 1-2, 482p.

Sick, H. 1997. Ornitologia Brasileira. Rio de Janeiro, Nova Fronteira. 862p.

Sick, H. 2001. Ornitologia Brasileira. Rio de Janeiro, Nova Fronteira. 912p.

Silveira, L.F. 2003. Filogenia dos Cracidae (Aves, Galliformes) com base em caracteres osteológicos. Tese (Doutorado). Universidade de São Paulo, Departamento de Zoologia, Instituto de Biociências. 212p.

Silveira, L.F.; Olmos, F. \& Long, A.J. 2004. Taxonomy, History and Status of the Alagoas Curassow Mitu mitu (Linnaeus, 1766), the world's most threatened Cracidae. Ararajuba, Revista Brasileira de Ornitologia, 12: 43-50.

Silveira, L.F.; Tomotani, B.M.; Cestari, C.; Straube, F.C. \& Piacentini, V.Q. 2017. Ortalis remota: a forgotten and critically endangered species of chachalaca (Galliformes: (racidae) from Eastern Brazil. Zootaxa, 4306: 524-536.

Snethlage, E. 1914. Catálogo das aves amazônicas: contendo todas as especies descriptas e mencionadas até 1913. Boletim do Museu Goeldi de Historia Natural e Ethnographia, 8: 1-530.

Temminck, J.C. 1815. Histoire Naturelle Généralle des Pigeons et des Gallinaces, Amsterdan, J.C.Sepp et Fils. 2 v.

Todd, W.E.C. 1932. Critical notes on the Cracidae. Proceedings of the Biological Society of Washington, 45: 209-214.

Vaurie, C. 1965. Systematic notes on the bird Family Cracidae: Ortalis guttata, Ortalis superciliaris, and Ortalis motmot. American Museum Novitates, 2232: 1-36.

Vaurie, C. 1968. Taxonomy of the Cracidae (Aves). Bulletin of the American Museum of Natural History, 138: 131-260.

Voisin, C.; Hennache, A. \& Voisin, J.F. 2015. List of type specimens of birds in the collections of the Muséum National d'Histoire Naturelle (Paris, France). 24. Cracidae, Odontophoridae, Phasianidae and Numididae. Journal of the National Museum (Prague), Natural History Series, 184: 1-42.

Wagler, J. 1830. Revisio generis Penelope. Isis von Oken, 35: 1109-1112.

Wagler, J. 1832. Neue Sippen und Gattungen der Säugethiere und Vögel. Isis von Oken, 3: 1218-1235.

Wiley, R.H. 2010. Alfonso Olalla and his family: the ornithological exploration of Amazonian Peru. Bulletin of the American Museum of Natural History, 343: 1-68. 


\section{APPENDIX 1}

Results of the individual analysis testing for the differences in size of bill (a-c), tarsus (d), wing (e) and tail (f) between the two taxa (and also testing for sex differences). Blue: O. motmot; red: O. ruficeps. Dark-coloured symbols: males; lightcoloured symbols: females.

a)

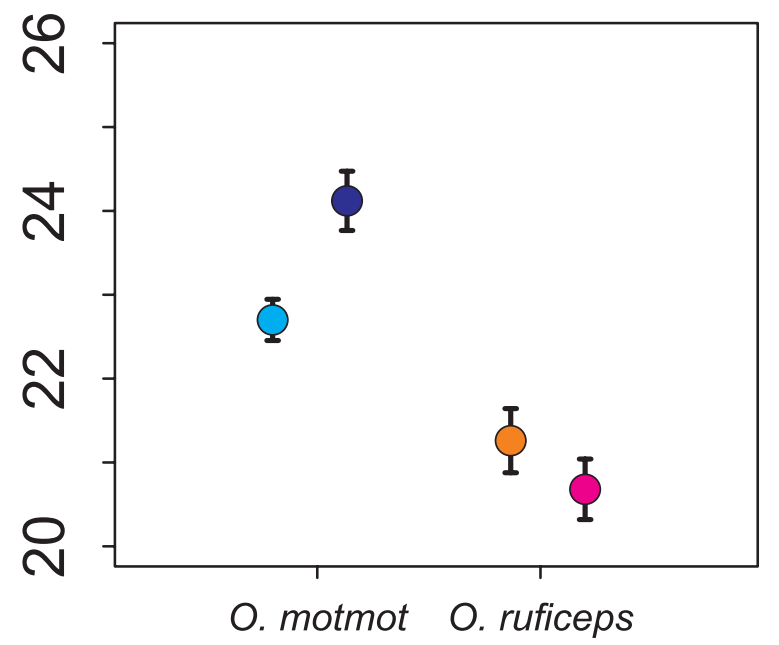

Bill culmen size ( $\mathrm{mm}$ )

c)

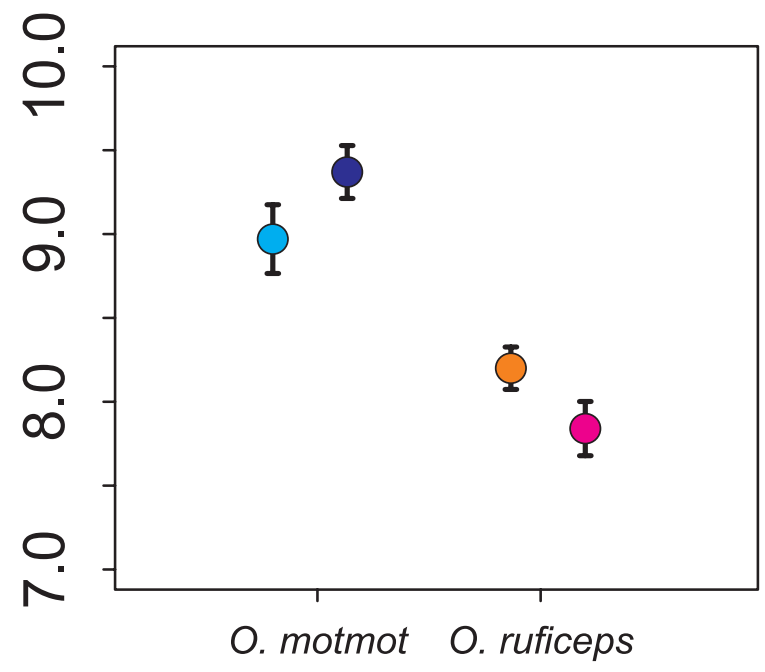

Bill width (mm)

e)

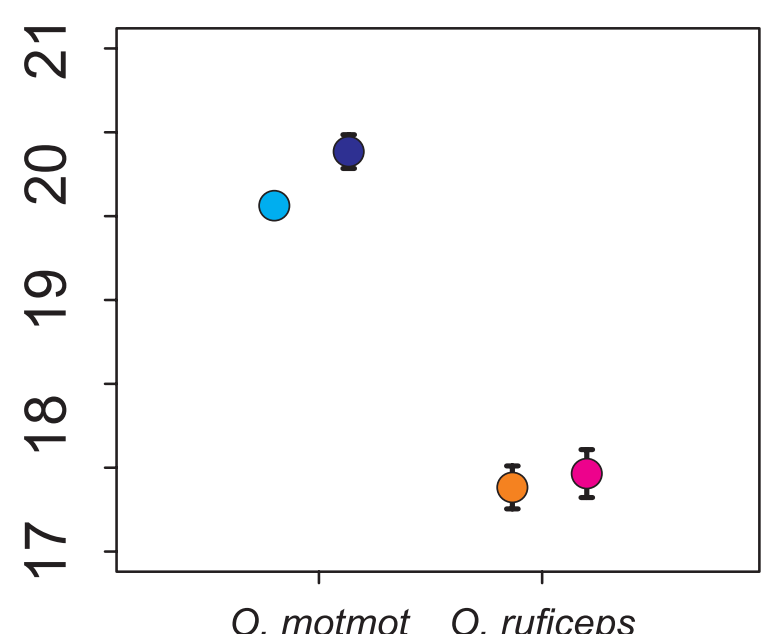

Wing size $(\mathrm{cm})$ b)

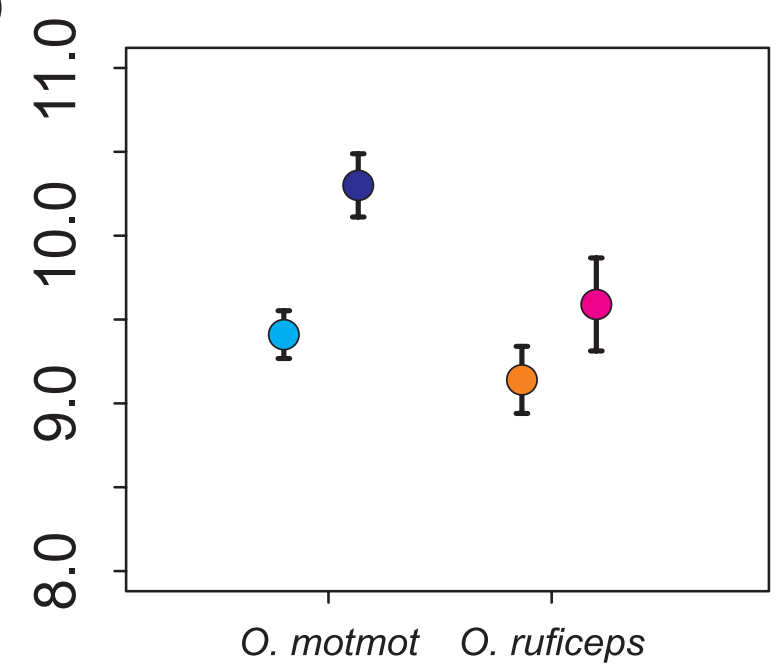

Bill depth ( $\mathrm{mm})$

d)

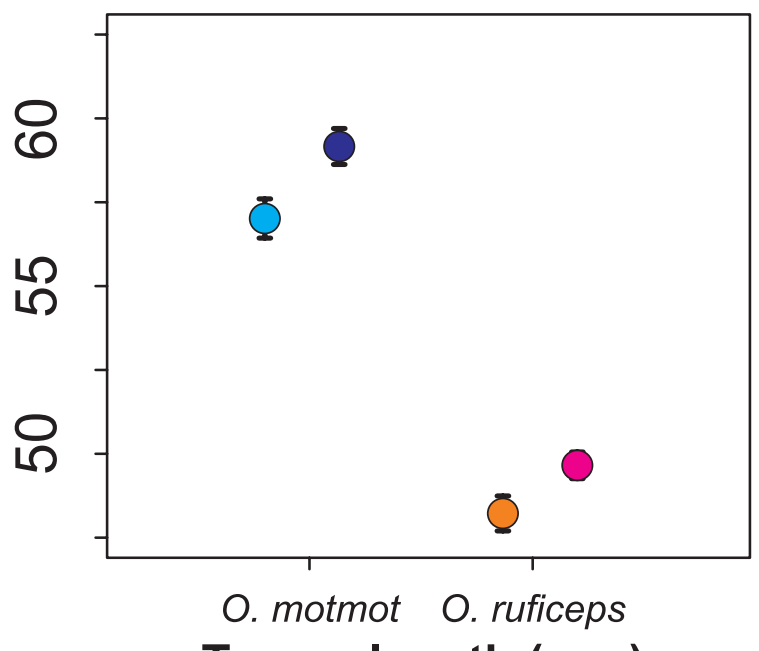

Tarsus length ( $\mathrm{mm}$ )

f)

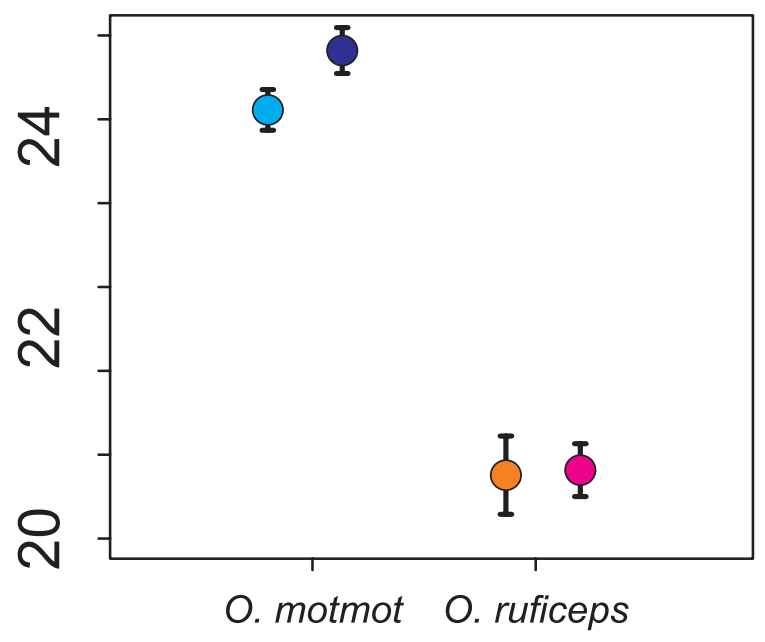

Tail size (cm) 


\section{APPENDIX 2}

Outcomes of the principal component analysis for morphometrics.

\begin{tabular}{lcccccc}
\hline & PC1 & PC2 & PC3 & PC4 & PC5 & PC6 \\
\hline Standard deviation & 1.90 & 0.84 & 0.67 & 0.54 & 0.37 & 0.36 \\
Proportion of Variance & 0.68 & 0.13 & 0.08 & 0.05 & 0.03 & 0.02 \\
Cumulative Proportion & 0.68 & 0.81 & 0.90 & 0.95 & 0.98 & 1.00 \\
Bill culmen & -0.42 & 0.47 & -0.52 & 0.32 & -0.27 & -0.40 \\
Bill depth & -0.20 & 0.60 & 0.75 & 0.05 & 0.12 & -0.09 \\
Bill width & -0.35 & 0.31 & -0.26 & -0.72 & 0.11 & 0.43 \\
Tarsus & -0.47 & -0.18 & -0.07 & 0.48 & 0.62 & 0.35 \\
Wing & -0.48 & -0.31 & 0.26 & 0.10 & -0.70 & 0.34 \\
Tail & -0.46 & -0.43 & 0.14 & -0.37 & 0.19 & -0.64 \\
\hline
\end{tabular}

\section{APPENDIX 3}

Outcomes of the principal component analysis for song.

\begin{tabular}{lcccccc}
\hline & PC1 & PC2 & PC3 & PC4 & PC5 & PC6 \\
\hline Standard deviation & 1.49 & 1.23 & 1.07 & 0.83 & 0.65 & 0.06 \\
Proportion of Variance & 0.37 & 0.25 & 0.19 & 0.12 & 0.07 & 0.00 \\
Cumulative Proportion & 0.37 & 0.62 & 0.81 & 0.93 & 1.00 & 1.00 \\
Total syllable length & 0.61 & -0.02 & -0.22 & -0.38 & 0.19 & 0.63 \\
Note 1 & -0.37 & -0.11 & -0.65 & -0.33 & 0.52 & -0.22 \\
Note 2 & 0.42 & 0.05 & -0.64 & 0.22 & -0.49 & -0.35 \\
Note 3 & 0.53 & -0.32 & 0.25 & 0.19 & 0.53 & -0.48 \\
Interval 1 & -0.02 & -0.67 & 0.15 & -0.58 & -0.41 & -0.17 \\
Interval 2 & 0.17 & 0.66 & 0.20 & -0.58 & -0.02 & -0.40 \\
\hline
\end{tabular}




\section{APPENDIX 4}

Results of the individual analysis testing for the differences in song and note duration between the two taxa. Blue: O. motmot, Red: O. ruficeps.

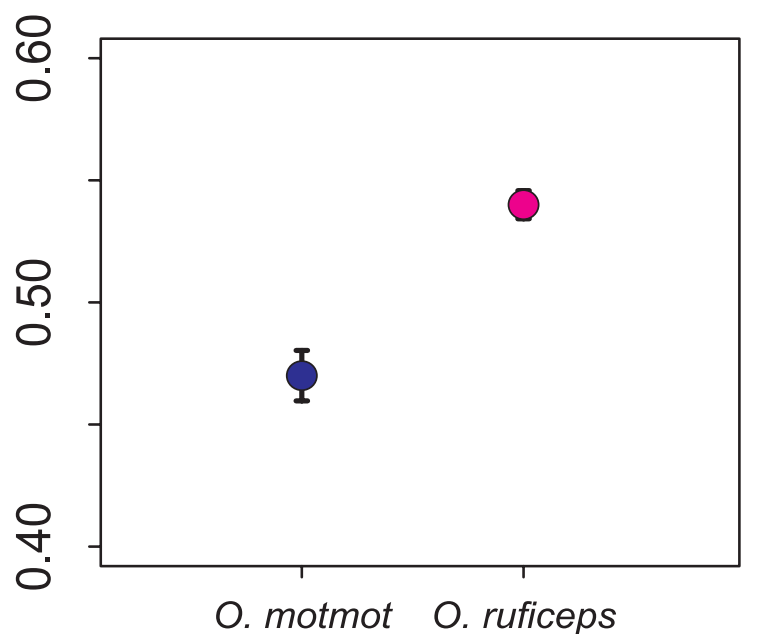

Total syllable length (s)
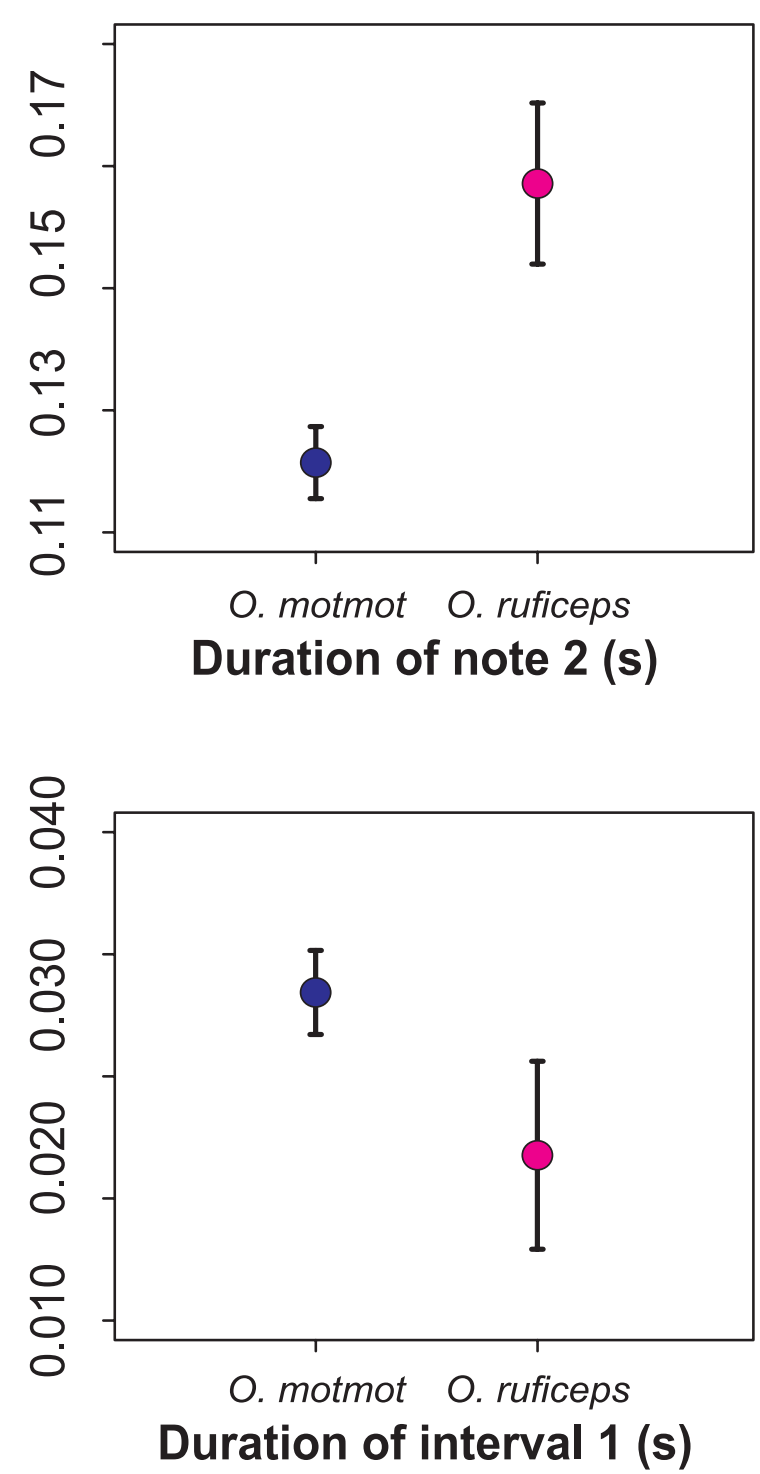

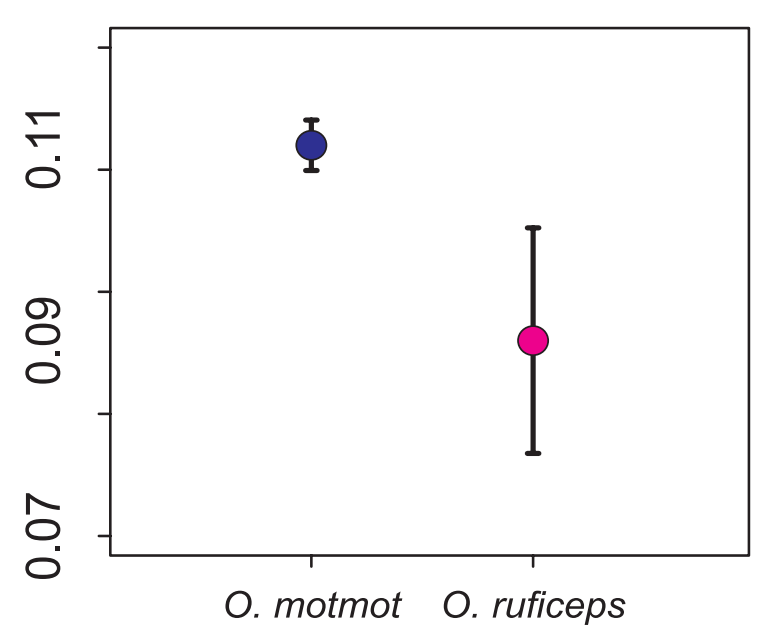

Duration of note 1 (s)

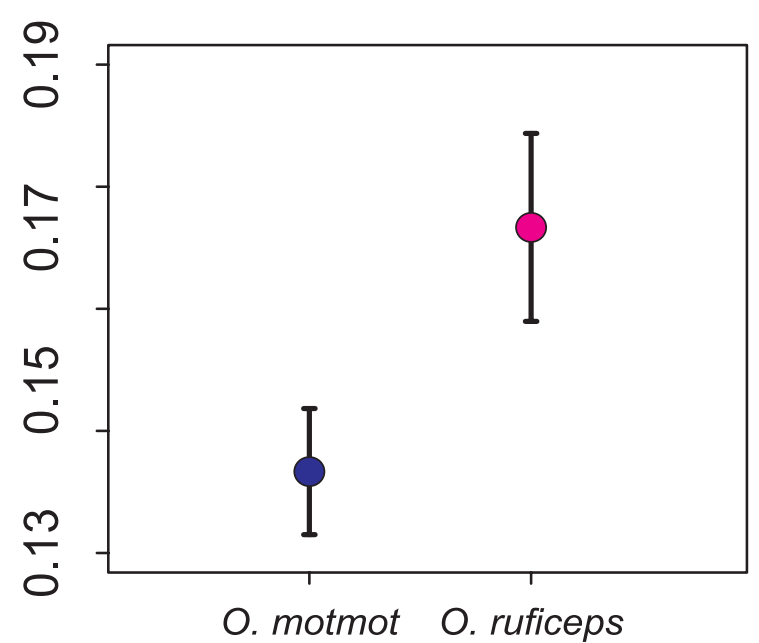

Duration of note 3 (s)

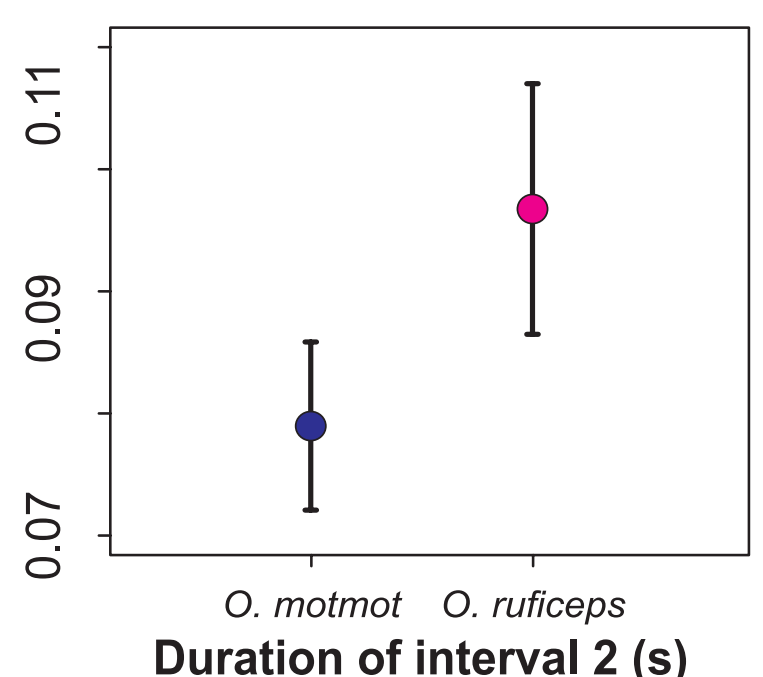

